\title{
Mass structure of hadrons and light-front sum rules in the ' $t$ Hooft model
}

\author{
Xiangdong Ji॰* \\ Department of Physics, University of Maryland, College Park, Maryland 20742, USA \\ Yizhuang $\mathrm{Liu}^{\dagger}$ \\ Tsung-Dao Lee Institute, Shanghai Jiao Tong University, Shanghai 200240, China \\ and Institut fur Theoretische Physik, Universitat Regensburg, D-93040 Regensburg, Germany \\ Ismail Zahed \\ Center for Nuclear Theory, Department of Physics and Astronomy, Stony Brook University, \\ Stony Brook, New York 11794-3800, USA
}

(Received 23 October 2020; accepted 19 March 2021; published 6 April 2021)

\begin{abstract}
We study the mass/energy structure of the bound state of hadrons in two-dimensional quantum chromodynamics in the large number of color limit (' $t$ Hooft model). We analyze separately the contributions from the traceless and trace part of the energy-momentum tensor, and show that the masses are related to the matrix elements of the scalar charge and Coulomb energy. We derive the light-front sum rules for the scalar charge and Coulomb energy, expressed in terms of the light-front wave functions, and find that they are regular at $x=0$ without the delta function contribution. We also consider the result for the massless Goldstone boson, as well as the structure of the gravitational form factors of the bound meson states.
\end{abstract}

DOI: $10.1103 /$ PhysRevD.103.074002

\section{INTRODUCTION}

One of the chief driving force for the future of the electron-ion collider (EIC) is the understanding of the origin of the nucleon mass [1]. There has been a lot of interest in understanding the mass structure following the original approach in Refs. [2,3] by studying the matrix elements of the energy-momentum tensor of quantum chromodynamics (QCD). A recent review can be found in [4], see also [5-9] for new developments. It is widely accepted, that its origin stems from the anomalous breaking of scale-invariance or the trace anomaly in quantum chromodynamics (QCD) which generates a nonperturbative mass scale, that is likely tied to the confinement of color and the spontaneous breaking of chiral symmetry in the QCD dynamics. A measure of the trace anomaly is captured by the matrix element of the gluon operator $F^{2}$, which is related to the twist-four gluon distribution function on the light cone [10]. Its first moment or the light-cone sum rule is related to the gluon matrix element in a nucleon state.

\footnotetext{
*xji@physics.umd.edu

†yizhuang.liu@sjtu.edu.cn

*ismail.zahed@stonybrook.edu
}

Published by the American Physical Society under the terms of the Creative Commons Attribution 4.0 International license. Further distribution of this work must maintain attribution to the author(s) and the published article's title, journal citation, and DOI. Funded by SCOAP.
The purpose of this paper is to shed some light on the origin of mass in two-dimensional spinor QCD, where fermionic and gluonic condensates develop in the vacuum state in the weak coupling regime and large $N_{c}$ [11], following on earlier investigations of the quasidistributions $[12,13]$ and lightfront mass sum rule [10]. In passing, we will briefly quote the result for two-dimensional QED. We will construct the corresponding chiral-odd quark and twist-four gluon distribution functions, and discuss their moments in relationship to the contribution to the mass/energy of the hadrons.

The organization of the paper is as follows: in Sec. II we provide an introduction to the model and its quantization in large $N_{c}$ limit. Both the equal-time quantization formalism and the light-front quantization formalism are introduced. In Sec. III, we introduce the symmetric energy-momentum tensor for two-dimensional QCD and discuss the mass sum rule in the rest frame. In Sec. IV we discuss the mass sum rule in the infinite momentum frame (IMF), and relates the quark and gluon contributions to light-front distribution functions. More specifically, in IV B we derive the chiral odd quark parton distribution function, and show that its zeroth moment is the scalar quark condensate or sigma term in a bound meson without the presence of a delta-function. We also show that the vacuum chiral condensate is directly expressible in terms of the would-be Goldstone parton distribution function. In Sec. IV C we derive the twistfour gluon parton distribution function in a bound meson state, and analyze its zeroth moment. In Sec. V we show that the energy momentum tensor in the bound meson is 
characterized by two-invariant form factors in relation to the graviton and dilaton form factors, suggesting the possibility of a direct measurement of the trace part of the energy-momentum tensor entering the mass sum rule in diffractive processes. In Sec. VI we derive a dual mass sum rule with the help of the colored axial-vector anomaly, where the mass budget is solely fermionic in any frame. Our conclusions are in Sec. VII. We briefly quote the results for massive two dimensional QED in Appendix A. Some additional aspects of the light cone wave functions in two dimensions are detailed in Appendix B, and a general virial theorem is derived in Appendix C.

\section{INTRODUCTION TO THE MODEL}

In this section we introduce the model, its quantization and its solution in the large $N_{c}$ limit. The Lagrangian of the theory is

$$
\mathcal{L}=-\frac{1}{4} F^{a \mu \nu} F_{\mu \nu}^{a}+\bar{\psi}(i \gamma \cdot D-m) \psi .
$$

Our space-time coordinates reads $(t, z)$, the metric is $(1,-1)$. We choose the Weyl-like basis $\gamma^{\mu}=\left(\sigma^{t},-i \sigma^{z}\right)$ with $\gamma^{5}=\gamma^{t} \gamma^{z}=\sigma_{3}$ where $\sigma_{i}$ are traditional Pauli matrices. The covariant-derivative reads $D^{\mu}=\partial^{\mu}-i g_{0} A^{a \mu} T^{a}$, modulo regularization. The color matrices are normalized to $\operatorname{Tr}\left(T^{a} T^{b}\right)=\delta^{a b} / 2$ with $a=1, \ldots, N_{c}^{2}-1$ and $\psi$ is in the fundamental representation. In two-dimensions, the gluonic contribution can be simplified using the observation that the anti-symmetric field strength relates to a gauge-covariant and colored pseudoscalar potential $E^{a}$

$$
F^{a \mu \nu}=\epsilon^{\mu \nu} E^{a} .
$$

In two dimensional $\mathrm{QCD}$, unlike the four dimensional version, the gauge-coupling $g_{0}$ has mass dimension equal to 1. The natural mass-scale in the gauge sector is setup by $m_{0}^{2}=\frac{g^{2} N_{c}}{2 \pi} \equiv \frac{\lambda}{2 \pi}$. For pure Yang-Mills in two dimensions, no dynamical degree of freedom such as the glueballs can be generated. The only intensive quantity with natural physical meaning is the string tension $\sigma=\pi m_{0}^{2} / 2$. When fermions are included, there is one more mass scale, the bare fermion mass $m$. The confining potential between color charges allows the formation of mesonic bound state, which can be exactly solved in the large $N_{c}$ limit. The bound-state equation for the mesons can either be derived in the $A^{z}=0$ axial gauge using equal-time quantization, or in the light-cone gauge using light-front quantization. It can be shown that by imposing the large meson momentum $P^{z} \rightarrow \infty$ limit on the equal-time wave function, one recovers the light-front wave functions $[12,13]$.

\section{A. Equal-time quantization in $A^{z}=0$ gauge}

We first introduce the equal-time quantization in the axial gauge, which was investigated first in Ref. [14]. See also Ref. [12] for a nice introduction. In this gauge, the equation of motion for the temporal component of the gauge field is nondynamical and can be expressed in terms of quark fields

$$
A^{0 a}=\frac{1}{\partial_{z}^{2}} g_{0} \bar{\psi} \gamma \gamma^{0} T^{a} \psi
$$

Thus, the pseudoscalar $E^{a}$ can be written as

$$
E^{a}=-\frac{1}{\partial_{z}} g_{0} \bar{\psi} \gamma^{0} T^{a} \psi
$$

The Hamiltonian reads in terms of the quark field

$$
\begin{aligned}
H= & \int d x \bar{\psi}\left(-\gamma^{z} \partial_{z}+m\right) \psi+\frac{g_{0}^{2}}{2} \int d z d z^{\prime}\left(\bar{\psi} \gamma^{0} T^{a} \psi\right)(z) \\
& \times \frac{1}{-\partial_{z}^{2}}\left(z-z^{\prime}\right)\left(\bar{\psi} \gamma^{0} T^{a} \psi\right)\left(z^{\prime}\right) .
\end{aligned}
$$

To diagonalize the Hamiltonian, let us introduce the dressed quark field

$\psi_{A}(z)=\int \frac{d p}{2 \pi} \frac{1}{\sqrt{2 E(p)}} e^{i p z}\left(u(p) a_{A}(p)+v(-p) d_{A}^{\dagger}(-p)\right)$

with $A=1, \ldots, N_{c}$. The anticommuting relations for the creation-annihilation operators are

$$
\begin{aligned}
{\left[a_{A}(p), a_{B}^{\dagger}\left(p^{\prime}\right)\right]_{+} } & =\left[b_{A}(p), b_{B}^{\dagger}\left(p^{\prime}\right)\right]_{+} \\
& =\delta_{A B}(2 \pi) \delta\left(p-p^{\prime}\right) .
\end{aligned}
$$

By writing the spinors as

$$
\begin{gathered}
u(p)=\sqrt{E(p)} e^{-\frac{\gamma^{z}}{2} \theta(p)}(1,1)^{T}, \\
v(-p)=\sqrt{E(p)} e^{-\frac{\gamma^{z}}{2} \theta(p)}(1,-1)^{T},
\end{gathered}
$$

and plugging into the Hamiltonian, one finds that there is no linear term in the creation-annihilation operators $a_{A}, b_{A}$ if $E(p)$ and $\theta(p)$ satisfy the gap equations

$$
\begin{aligned}
E(p)= & m \cos \theta(p)+p \sin \theta(p) \\
& +\frac{m_{0}^{2}}{4} \int_{\mathrm{PV}} \frac{\cos [\theta(p)-\theta(k)]}{(p-k)^{2}} \\
p \cos \theta(p)-m \sin \theta(p)= & \frac{m_{0}^{2}}{4} \int_{\mathrm{PV}} \frac{\sin [\theta(p)-\theta(k)]}{(p-k)^{2}} .
\end{aligned}
$$

The gap equation has no analytic solution in general. Nevertheless, one can show that the function $\theta(p)$ is odd in $p$, and $\theta(p) \rightarrow \pm \frac{\pi}{2}$ as $p \rightarrow \pm \infty$. One also needs 
the relation $\tan \theta(p) \rightarrow \frac{p}{m}+\mathcal{O}\left(\frac{1}{p}\right)$ as $p \rightarrow \infty$. In this case, one can diagonalize the Hamiltonian in the large $N_{c}$ limit in the form

$$
H=\int \frac{d P}{2 \pi} \sum_{n} E_{n}(P) m_{n}^{\dagger}(P) m_{n}(P)+\mathcal{O}\left(\frac{1}{N_{c}}\right)
$$

where $E_{n}=\sqrt{P^{2}+M_{n}^{2}}$ is the energy of the meson, and the creation-annihilation operator of the meson state reads

$$
\begin{aligned}
m_{n}(P)= & \int \frac{d k}{\sqrt{2 \pi N_{c}|P|}} \times \sum_{A}\left(b_{A}(P-k) a_{A}(k) \phi_{n}^{+}(k, P)\right. \\
& \left.+a_{A}^{\dagger}(k-P) b_{A}^{\dagger}(-k) \phi_{n}^{-}(k, P)\right),
\end{aligned}
$$

provided that the wave functions $\phi_{n}^{ \pm}$satisfy the Bars-Green equation [14]

$$
\begin{aligned}
& \left(E(p)+E(P-p) \mp E_{n}(p)\right) \phi_{n}^{ \pm}(p, P) \\
& =\frac{m_{0}^{2}}{2} \int_{\mathrm{PV}} \frac{d k}{(p-k)^{2}}\left(C_{P}(p, k) \phi_{n}^{ \pm}(k, P)\right. \\
& \left.\quad-S_{P}(p, k) \phi_{n}^{\mp}(k, P)\right),
\end{aligned}
$$

with

$$
\begin{aligned}
& C_{P}(p, k)=\cos \left(\frac{\theta(p)-\theta(k)}{2}\right) \cos \left(\frac{\theta(P-p)-\theta(P-k)}{2}\right) \\
& S_{P}(p, k)=\sin \left(\frac{\theta(p)-\theta(k)}{2}\right) \sin \left(\frac{\theta(P-p)-\theta(P-k)}{2}\right)
\end{aligned}
$$

The $m_{n}(P)$ satisfy the commutation relations

$$
\left[m_{n}(P), m_{n^{\prime}}^{\dagger}\left(P^{\prime}\right)\right]=2 \pi \delta_{n n^{\prime}} \delta\left(P-P^{\prime}\right),
$$

provided that the $\phi_{n}^{ \pm}$are normalized as

$$
\int d k \phi_{n}^{+}(k, P) \phi_{n^{\prime}}^{+}(k, P)-\phi_{n}^{-}(k, P) \phi_{n^{\prime}}^{-}(k, P)=|P| \delta_{n, n^{\prime}} .
$$

The vacuum state is defined by $m_{n}(P)|0\rangle=0$, which is a coherent state in terms of the original quark creationannihilation operators. The meson state is given by

$$
|P, n\rangle=\sqrt{2 E_{n}} m_{n}^{\dagger}(P)|0\rangle .
$$

The original meson-quark-antiquark operator can be reconstructed as

$$
\begin{aligned}
\frac{1}{\sqrt{N_{c}}} \sum_{A} b_{A}(P-k) a_{A}(k)= & \sqrt{\frac{2 \pi}{|P|}} \sum_{n}\left(\phi_{n}^{+}(k, P) m_{n}(P)\right. \\
& \left.-\phi_{n}^{-}(k-P,-P) m_{n}^{\dagger}(-P)\right)
\end{aligned}
$$

As $P \rightarrow \infty$, the backward moving component $\phi_{n}^{-} \rightarrow 0$ at a rate of $\frac{1}{P^{2}}$, while the forward component approaches the light-front wave function $\phi_{n}^{+}(k, P) \rightarrow \phi_{n}\left(\frac{k}{P}\right)$ and is only supported in the interval $0<k<P$. The same light-front wave function can also be obtained from light-cone quantization which will be introduced next.

\section{B. Light-front quantization in $A^{+}=0$ gauge}

Here we present an introduction to the light-front quantization of two-dimensional QCD and derive the ' $t$ Hooft equation for the light-front wave functions. Our convention for light-front coordinates are $x^{ \pm}=\frac{1}{2}(t \pm z)$. In the light cone gauge $A_{-}=A^{+}=0$, the Lagrangian is

$$
\begin{aligned}
\mathcal{L}_{1+1}= & \frac{1}{8}\left(\partial^{+} A^{a-}\right)^{2}+\psi_{+}^{\dagger} i D^{-} \psi_{+}+\psi_{-}^{\dagger} i \partial^{+} \psi_{-} \\
& +i m\left(\psi_{+}^{\dagger} \psi_{-}-\psi_{-}^{\dagger} \psi_{+}\right)
\end{aligned}
$$

in the Weyl-like basis with $\gamma^{5} \psi_{ \pm}= \pm \psi_{ \pm}$. In this gauge $\psi_{-}=\left(m / \partial^{+}\right) \psi_{+}$is a constraint field, and $A^{-}$can be eliminated by its equation of motion. The corresponding Hamiltonian on the light front with $x^{+}=0$, follows canonically in the form

$$
\begin{aligned}
P^{-}= & \int \frac{1}{2}\left(E^{a} E^{a}+m \bar{\psi} \psi\right) d y^{-} \\
= & -\frac{i m^{2}}{2} \int d x^{-} d y^{-} \psi_{+}^{\dagger}\left(x^{-}\right) \theta\left(y^{-}-x^{-}\right) \psi_{+}\left(y^{-}\right) \\
& -\frac{g^{2}}{4} \int d x^{-} d y^{-} \psi_{+}^{\dagger} T^{a} \psi_{+}\left(x^{-}\right)\left|x^{-}-y^{-}\right| \psi_{+}^{\dagger} T^{a} \psi_{+}\left(y^{-}\right) .
\end{aligned}
$$

The free field reads

$$
\psi_{+A}\left(z^{-}\right)=\int_{0}^{\infty} \frac{d k^{+}}{2 \pi}\left(e^{-i k^{+} z^{-}} a_{A}\left(k^{+}\right)+e^{+i k^{+} z^{-}} b_{A}^{\dagger}\left(k^{+}\right)\right)
$$

and the anticommuting rules for the creation-annihilation operators are

$$
\begin{aligned}
{\left[a_{A}\left(k^{+}\right), a_{B}^{\dagger}\left(p^{+}\right)\right]_{+} } & =\left[b_{A}\left(k^{+}\right), b_{B}^{\dagger}\left(p^{+}\right)\right]_{+} \\
& =\delta_{A B}(2 \pi) \delta\left(k^{+}-p^{+}\right) .
\end{aligned}
$$

The bound states associated to (20) are eigenstates to $P^{-}|P, n\rangle=P_{n}^{-}|P, n\rangle$, subject to Gauss law (color singlet). In the large $N_{c}$ limit and weak coupling with $m \gg m_{0}$ pair production is suppressed and mesons and baryons are 
stable. In leading order in $1 / N_{c}$, a meson with a pair of $q \bar{q}$ is represented by the colorless state

$$
|P, n\rangle=\int_{0}^{1} \frac{P^{+} d x}{\sqrt{\pi}} \frac{\varphi_{n}(x)}{\sqrt{N_{c}}} a_{A}^{\dagger}\left(x P^{+}\right) b_{A}^{\dagger}\left((1-x) P^{+}\right)|0\rangle
$$

with Feynman $x=k^{+} / P^{+}$such that $0 \leq x \leq 1$ and $x+\bar{x}=1$.

In this construction, it is not difficult to show that the amplitudes $\varphi_{n}(x)$ solve the ' $t$ Hooft equation [11]

$$
\frac{m^{2}}{x \bar{x}} \varphi_{n}(x)-m_{0}^{2} \int_{\mathrm{PV}} d y \frac{\varphi_{n}(y)-\varphi_{n}(x)}{(x-y)^{2}}=M_{n}^{2} \varphi_{n}(x),
$$

with the canonical mass scale $m_{0}^{2}=\lambda / \pi$ and $\lambda=g^{2} N_{c}$ the ' $t$ Hooft coupling. If we take the large $P$ limit in the BarsGreen equation Eq. (13), then we obtain the same ' $t$ Hooft equation here for $\phi_{n}^{+}(k) \rightarrow \varphi_{n}(x)$ where $x=\frac{k}{P}$. Thus, by taking the large momentum limit of the equal-time wave function we recover the light-front wave function.

\section{HADRON MASS/ENERGY SUM RULE IN EQUAL-TIME QUANTIZATION}

To study the mass structure of a hadron, one can start from the energy momentum tensor of the theory [2]. Massive spinor QCD is characterized by the symmetric energy momentum tensor

$$
\begin{aligned}
T^{\mu \nu} & =\frac{2}{\sqrt{-g}} \frac{\delta S_{1+1}}{\delta g_{\mu \nu}} \\
& \left.=F^{a \mu \lambda} F_{\lambda}^{a \nu}+\frac{1}{4} g^{\mu \nu} F^{2}+\frac{1}{2} \bar{\psi} \gamma^{[\mu} i \stackrel{\leftrightarrow}{D}\right]^{]_{+}} \psi
\end{aligned}
$$

with $\stackrel{\leftrightarrow}{D}=\vec{D}-\stackrel{\leftarrow}{D}$ and []$_{+}$denotes symmetrization. In terms of $E^{a}$, the stress tensor reads

$$
\left.T^{\mu \nu}=\frac{1}{2} g^{\mu \nu} E^{a} E^{a}+\frac{1}{2} \bar{\psi} \gamma^{[\mu} i \stackrel{\leftrightarrow}{D}\right]_{+} \psi
$$

It is conserved $\partial_{\mu} T^{\mu \nu}=0$, with a non-vanishing trace

$$
T_{\mu}^{\mu}=E^{a} E^{a}+m \bar{\psi} \psi
$$

In two-dimensions, QCD is super-renormalizable, hence non-conformal. Amusingly, the trace part in (27) resembles the trace part of QCD with a dimensionless coupling in four dimensions [2].

\section{A. Virial theorem}

The energy-momentum tensor can be decomposed as the sum of a traceless and trace part [2]

$$
T^{\mu \nu}=\hat{T}^{\mu \nu}+\frac{1}{2} g^{\mu \nu} T_{\alpha}^{\alpha},
$$

where the traceless part reads

$$
\left.\hat{T}^{\mu \nu}=\frac{1}{2} \bar{\psi} \gamma^{[\mu} i \stackrel{\leftrightarrow}{D}\right]_{+} \psi-\frac{g^{\mu \nu}}{2} m \bar{\psi} \psi
$$

and the trace part is given in Eq. (27).

For a single-particle state $|P, n\rangle$ with the standard normalization $\left\langle P, n \mid P^{\prime}, n^{\prime}\right\rangle=2 E_{n} \delta_{n, n^{\prime}}(2 \pi) \delta\left(P-P^{\prime}\right)$, one has

$$
\left\langle P, n\left|T^{\mu \nu}\right| P, n\right\rangle=2 P^{\mu} P^{\nu} .
$$

By considering the 00 component in the rest frame, one finds the relation between the matrix elements

$$
\begin{aligned}
& \left\langle 0, n\left|\bar{\psi}\left(-i \gamma^{z} D_{z}\right) \psi\right| 0, n\right\rangle+\langle 0, n|m \bar{\psi} \psi| 0, n\rangle \\
& \quad+\left\langle 0, n\left|\frac{1}{2} E^{a} E^{a}\right| 0, n\right\rangle=2 M_{n}^{2}
\end{aligned}
$$

By taking the trace, one has in any frame

$$
\left\langle P, n\left|T_{\mu}^{\mu}\right| P, n\right\rangle=\left\langle P, n\left|m \bar{\psi} \psi+E^{a} E^{a}\right| P, n\right\rangle=2 M_{n}^{2} .
$$

which basically sets the scales for the theory.

We note that even though two-dimensional QCD is superrenormalizable, the individual operators can still diverge logarithmically. However, since $T^{\mu \nu}$ is renormalization free (conserved), the same holds for its trace (27) and its time-component $T^{00}$ in (31). In addition $m \bar{\psi} \psi$ is also renormalization free, so both $E^{a} E^{a}$ and $\bar{\psi}\left(i \gamma^{z} D_{z}\right) \psi$ are UV safe. As a result, the mass decomposition in (31) is well defined, and the quantities $\mathcal{M}, \mathcal{G}, \mathscr{G}$ defined in (38) below, are all scheme and scale independent, in contrast to the corresponding situation in four-dimensional QCD.

With this in mind, lets us consider the mass sum rule in the rest frame. The Hamiltonian can be obtained from the $T^{00}$ component

$$
\begin{aligned}
H & =\hat{H}+\bar{H} \\
& =\int d z\left(\bar{\psi} i \vec{\gamma} \cdot \vec{D} \psi+m \bar{\psi} \psi+\frac{1}{2} E^{a} E^{a}\right),
\end{aligned}
$$

where the traceless part $\hat{H}$ and the trace part $\bar{H}$ read

$$
\begin{aligned}
& \hat{H}=\int d z \hat{T}^{00}=\int d z \bar{\psi}\left(-i \gamma^{z} D_{z}+\frac{1}{2} m\right) \psi, \\
& \bar{H}=\int d z \frac{1}{2} T_{\mu}^{\mu}=\int d z\left(\frac{1}{2} E^{a} E^{a}+\frac{1}{2} m \bar{\psi} \psi\right) .
\end{aligned}
$$

If we define the average

$$
\langle O\rangle_{P, n}=\frac{\langle P, n|O| P, n\rangle}{\langle P, n \mid P, n\rangle}
$$


and notice that $\int d z=L=2 \pi \delta_{p}(0)$, we can read from Eq. (32) that the contribution of the trace part to the total mass in the rest frame is half of the mass, which is the relativistic virial theorem first discussed in Ref. [2]

$$
\begin{aligned}
\langle\bar{H}\rangle_{0, n} & =\frac{M_{n}}{2}, \\
\langle\hat{H}\rangle_{0, n} & =\frac{M_{n}}{2} .
\end{aligned}
$$

More explicitly, one obtains the mass-sum rule

$$
\begin{aligned}
\langle\bar{H}\rangle_{0, n} & =\mathcal{G}+\frac{1}{2} \mathcal{M}=\frac{M_{n}}{2} \\
\langle\hat{H}\rangle_{0, n} & =\mathcal{K}+\frac{1}{2} \mathcal{M}=\frac{M_{n}}{2} .
\end{aligned}
$$

or more succinctly,

$$
\mathcal{K}+\mathcal{G}+\mathcal{M}=M_{n}
$$

with

$$
\begin{aligned}
\mathcal{M} & =\left\langle\int d z m \bar{\psi} \psi\right\rangle_{0, n}=\frac{\langle 0, n|m \bar{\psi} \psi| 0, n\rangle}{2 M_{n}} \\
\mathcal{K} & =\left\langle\int d z \bar{\psi}\left(-i \gamma^{z} D_{z}\right) \psi\right\rangle_{0, n}=\frac{\left\langle 0, n\left|\bar{\psi}\left(-i \gamma^{z} D_{z}\right) \psi\right| 0, n\right\rangle}{2 M_{n}} \\
\mathcal{G} & =\left\langle\int d z \frac{1}{2} E^{a} E^{a}\right\rangle_{0, n}=\frac{\left\langle 0, n\left|\frac{1}{2} E^{a} E^{a}\right| 0, n\right\rangle}{2 M_{n}}
\end{aligned}
$$

Comparing the two equations in Eq. (36), one obtains in the rest frame

$$
\mathcal{K}=\mathcal{G}
$$

The fermion kinetic and gluon energies in the rest frame are equal. This is consistent with the virial theorem $\langle|p|\rangle=$ $\left\langle r \frac{\partial V}{\partial r}\right\rangle$ for a relativistic quantum mechanical system [15], with the Hamiltonian $H=|p|+V(r)$ and a linear potential $V(r)=\sigma r$, if the gluon energy is identified with the average of the linear potential energy. This is also consistent with the results in Ref. [16] for massive state $\bar{s} s, \bar{c} c$ and in the $m \rightarrow \infty$ limit. This can also be obtained from a Feynman-Hellman theorem argument which is given in Appendix C.

\section{B. Partonic contributions to $\mathcal{G}, \mathcal{K}, \mathcal{M}$}

We now evaluate explicitly the matrix-elements above. We start with the scalar matrix element $\langle P, n|m \bar{\psi} \psi| P, n\rangle$. It can be calculated in a generic frame in terms of the wave functions $\phi_{n}^{ \pm}$in Eq. (13) as

$$
\begin{aligned}
\langle P, n|m \bar{\psi} \psi| P, n\rangle= & \frac{2 m E_{n}(P)}{|P|} \times \int d p[\cos \theta(p)+\cos \theta(\bar{p})] \\
& \times\left[\left|\phi_{n}^{+}\right|^{2}+\left|\phi_{n}^{-}\right|^{2}\right](k, P) .
\end{aligned}
$$

Since the matrix element is independent of $P$, by taking the $P \rightarrow \infty$ limit one obtains in terms of the light-front wave function in Eq. (24)

$$
\langle P, n|m \bar{\psi} \psi| P, n\rangle=2 m^{2} \int_{0}^{1} d x \frac{\varphi_{n}^{\dagger}(x) \varphi_{n}(x)}{x \bar{x}} .
$$

Therefore

$$
\mathcal{M}=\frac{m^{2}}{M_{n}} \int_{0}^{1} d x \frac{\varphi_{n}^{\dagger}(x) \varphi_{n}(x)}{x \bar{x}},
$$

gives the quark mass contribution.

Similarly, for the gluonic contribution one obtains in term of the light-front wave function

$$
\begin{aligned}
\langle P, & \left.n\left|\frac{1}{2} E^{a} E^{a}\right| P, n\right\rangle \\
& =-m_{0}^{2} \mathrm{PV} \int_{0}^{1} d x d y \frac{\varphi_{n}^{\dagger}(x)\left(\varphi_{n}(y)-\varphi_{n}(x)\right)}{(x-y)^{2}},
\end{aligned}
$$

and

$$
\mathcal{G}=-\frac{m_{0}^{2}}{2 M_{n}} \mathrm{PV} \int_{0}^{1} d x d y \frac{\varphi_{n}^{\dagger}(x)\left(\varphi_{n}(y)-\varphi_{n}(x)\right)}{(x-y)^{2}} .
$$

which is again frame-independent.

The kinetic energy contribution in the rest frame can be calculated as

$$
\begin{aligned}
& \left\langle P, n\left|\bar{\psi}\left(-i \gamma^{z} D_{z}\right) \psi\right| P, n\right\rangle \\
& =\frac{2 E_{n}(P)}{|P|} \times \int d p[p \sin \theta(p)+\bar{p} \sin \theta(\bar{p})] \\
& \quad \times\left[\left|\phi_{n}^{+}\right|^{2}+\left|\phi_{n}^{-}\right|^{2}\right](p, P) .
\end{aligned}
$$

By using the virial theorem in Eq. (39), one can show that the kinetic energy in the rest frame is equal to

$$
\mathcal{K}=-\frac{m_{0}^{2}}{2 M_{n}} \mathrm{PV} \int_{0}^{1} d x d y \frac{\varphi_{n}^{\dagger}(x)\left(\varphi_{n}(y)-\varphi_{n}(x)\right)}{(x-y)^{2}} .
$$

It is nontrivial to prove this using the Bars-Green equation directly. However, using the bosonized Hamiltonian in the large $N_{c}$ limit, it can be shown directly that $\mathcal{G}=\mathcal{K}$. Using the explicit form of the 't Hooft equation, it is easy to see that Eq. (36) is satisfied.

To summarize, in the rest frame, the mass of a hadron in two dimensional QCD comes from three sources: the quark kinetic energy $\mathcal{K}$, the gluon potential energy $\mathcal{G}$, and finally 
the quark mass $\mathcal{M}$. The first two are equal with $\mathcal{K}=G$. In Sec. V, we show how the gluonic content of the hadron mass can be related to experimentally measurable quantities.

\section{Relation to an observable}

To relates the mass sum rule to an observable, one may consider the contribution from the traceless part of the energy-momentum tensor, which is a twist-two operator. The light-front sum rule, can relate the $T^{++}$component of the stress tensor to the twist-two parton distribution function. As shown in Ref. [16], $\frac{1}{2} T^{++}$dominates the traceless part $\hat{T}^{00}$ of $T^{00}$ in the large momentum frame

$$
\left\langle P, n\left|\bar{\psi}\left(-i \gamma^{z} D_{z}+\frac{m}{2}\right) \psi\right| P, n\right\rangle \rightarrow\left(P^{+}\right)^{2}+\mathcal{O}\left(\frac{1}{P^{+}}\right) .
$$

In two dimensions, there is only one quark twist-two operator and no gluon twist-two operator as there is no dynamical gluon which can carry the hadron momentum. One can define the quark parton distribution function(PDF) for the meson state

$$
q_{n}(x)=\int \frac{d x^{-}}{4 \pi} e^{-i x^{-} P^{+} x}\left\langle P, n\left|\bar{\psi}\left(x^{-}\right) \gamma^{+}\left[x^{+}, 0\right] \psi(0)\right| P, n\right\rangle .
$$

It can be calculated straightforwardly as

$$
q_{n}(x)=\left|\varphi_{n}(x)\right|^{2} .
$$

The first moment of the quark-PDF is related to the traceless part of $T^{\mu \nu}$ through

$$
\left\langle P, n\left|T^{++}\right| P, n\right\rangle=4\left(P^{+}\right)^{2} \int_{0}^{1} d x x q_{n}(x) .
$$

This is consistent with the normalization

$$
\left\langle P, n\left|T^{++}\right| P, n\right\rangle=2\left(P^{+}\right)^{2},
$$

since one has

$$
\int_{0}^{1} d x x q_{n}(x)=\frac{1}{2} \int_{0}^{1}(x+1-x)\left|\varphi_{n}(x)\right|^{2}=\frac{1}{2}
$$

due to the relation $\varphi_{n}(1-x)=(-1)^{n} \varphi_{n}(x)$. Therefore, the twist-two light-front sum rule does not provide an additional information other than that the kinetic energy $\mathcal{K}$ of the quark plus half the quark mass term $\frac{1}{2} \mathcal{M}$ contribute half of the meson mass.

\section{IMF SUM RULE AND LIGHT-FRONT DISTRIBUTION FUNCTIONS}

Let us consider the mass-sum rule in the IMF. The lightcone Hamiltonian is related to the $T^{+-}$component of the stress tensor

$$
\begin{aligned}
T^{+-} & =\frac{1}{2} E^{a} E^{a}+\frac{1}{2} \bar{\psi} i \gamma \cdot D \psi \\
& =\frac{1}{2} E^{a} E^{a}+\frac{1}{2} m \bar{\psi} \psi,
\end{aligned}
$$

where in the second line, the equation of motion has been used. The mass sum-rule in the light front then reads

$P^{-}=\frac{M_{n}^{2}}{2 P^{+}}=\left\langle\int d x^{-} \frac{1}{2} m \bar{\psi} \psi\right\rangle_{P, n}+\left\langle\int d x^{-} \frac{1}{2} E^{a} E^{a}\right\rangle_{P, n}$.

which is

$$
2 \mathcal{G}+\mathcal{M}=M_{n},
$$

by using Eq. (38) and the normalization $\langle P \mid P\rangle=P^{+} L^{-}$ where $L^{-}=\int d x^{-}$is the length along the light-cone spatial direction $x^{-}$. Here both terms are higher-twist [10] where the twist are defined as the mass dimension minus the longitudinal spin. This is consistent with the light-cone sum-rule in Ref. [16]. We now discuss how the light-cone mass sum-rule can be related to moments of twist-three and twist-Four gluon and quark distributions functions. This will help understand the possible zero-mode contributions at $x=0$.

\section{A. Fermionic and gluonic distributions}

The fermionic mass contribution to the mass sum rule is directly probed by the chiral-odd scalar quark bilocal (twist-three light-front distribution) [10]

$Q_{n}(x)=\frac{P_{n}^{+}}{2 M_{n}^{2}} \int \frac{d z}{2 \pi} e^{i x P_{n}^{+} z}\langle P, n|m \bar{\psi}(0)[0, z] \psi(z)| P, n\rangle$

with the zeroth moment fixed

$$
Q_{n, 0}=\int d x Q(x)=\frac{\langle P, n|m \bar{\psi} \psi| P, n\rangle}{2 M_{n}^{2}}
$$

The gluon mass contribution can be probed by the twistfour gluon gauge-invariant bi-local

$$
\begin{aligned}
F_{n}(x) & =\frac{P_{n}^{+}}{4 M_{n}^{2}} \int \frac{d z}{2 \pi} e^{i x P_{n}^{+} z}\left\langle P, n\left|F_{\mu \nu}(0)[0, z] F^{\mu \nu}(z)\right| P_{n}\right\rangle \\
& =\frac{P_{n}^{+}}{2 M_{n}^{2}} \int \frac{d z}{2 \pi} e^{i x P_{n}^{+} z}\langle P, n|E(0)[0, z] E(z)| P, n\rangle,
\end{aligned}
$$


with the zeroth moment

$$
F_{n, 0}=\int d x F_{n}(x)=\frac{\left\langle P, n\left|E^{a} E^{a}\right| P, n\right\rangle}{2 M_{n}^{2}} .
$$

The light-front mass-sum rule then reads

$$
Q_{n, 0}+F_{n, 0}=1 \text {. }
$$

Below we discuss each of these two distribution functions in detail, particularly the possible existence of a distribution $\delta(x)$ which may ruin the practical sum rule.

\section{B. Chiral-odd quark parton distribution and light-cone sum rule}

In light-front quantization, zero modes are important. In fact, the chiral condensate in the vacuum comes entirely from zero modes. It has been suggested that $Q_{n}(x)$ has a regular and singular part [17]

$$
Q_{n}(x)=\delta(x) Q_{n, 0}+Q_{n, \text { reg }}(x)
$$

provided that the zeroth moment of the regular part vanishes. The delta-function maybe viewed as a signal of the quark condensate on the light cone. Its possible presence affects the partonic sum rules, a point of recent emphasis $[10,18,19]$.

\section{Analysis in light cone gauge}

Equation (55) can be readily calculated in the light cone gauge using the canonical formulation briefly reviewed before. More specifically, in the light cone gauge

$$
\begin{aligned}
\bar{\psi}(0)[0, z] \psi(z)= & \psi_{+}^{\dagger}(0)\left(-i \psi_{-}\right)(z)+\left(-i \psi_{-}\right)^{\dagger}(0) \psi_{+}(z) \\
= & \psi_{+}^{\dagger}(0)\left(-i\left(m / \partial^{+}\right) \psi_{+}\right)(z) \\
& +\left(-i\left(m / \partial^{+}\right) \psi_{+}\right)^{\dagger}(0) \psi_{+}(z)
\end{aligned}
$$

The fermionic field in (61) is given in (21). The fact that the expectation value in (61) is multiplied by $m$ is natural, since the chiral odd operator $\bar{\psi} \psi$ creates a left-right pair which in the light cone and $m=0$ cannot interact. It is however uncorrect to conclude that the matrix element vanishes as $m \rightarrow 0$ as we now show.

Inserting (61) into (55), and using the bound meson wave function (23) in leading order in $1 / N_{c}$, lead to a string of contractions in the vacuum. A repeated use of (22) yields the result

$$
Q_{n}(x)=\frac{2 m}{M_{n}} \frac{\left|\varphi_{n}(x)\right|^{2}}{x}
$$

In agreement with the Weisberger relation for the parton model in four dimensions [20,21]. We have made explicit the index- $n$ for an $n$-bound meson state. The occurrence of the overall factor of $m$ reflects on the chiral odd character of the bilocal operator. The zeroth moment of (62) is dominated by the singular behavior of the parton distribution funtion near the edge in (B3), i.e., $\varphi_{n}(x) \sim C_{n}(x \bar{x})^{\beta}$ as reviewed in Appendix B. The result is finite

$$
Q_{n, 0}=\frac{2 m}{M_{n}} \int_{0}^{1} d x \frac{\left|\varphi_{n}(x)\right|^{2}}{x}=\frac{C_{n}^{2} \pi m_{0}}{M_{n} \sqrt{3}}
$$

even in the chiral limit. A comparison with (56) implies the scalar condensate in the $n$-bound state

$$
\langle P, n|\bar{\psi} \psi| P, n\rangle=C_{n}^{2} \frac{2 \pi m_{0}}{\sqrt{3}}=-4 \pi C_{n}^{2} \frac{\langle\bar{\psi} \psi\rangle}{N_{c}}
$$

The last identity makes use of (66) below.

While the finite sum rule (56) is fulfilled for all bound states, the chiral odd quark distribution (62) does not develop a delta-function. Rather, the rapid vanishing of the wave function or parton distribution amplitude (PDA) at the edge with the exponent $\beta \sim m$ is what enforces the sum rule. In retrospect, this is expected since the chiral odd character of the operator generates a pre-factor $m$ that requires compensation for a finite zeroth moment. In a way, the vacuum physics is encoded in the $x \sim 0$ region of the chiral odd parton distribution function, not in the way it diverges but in the way it vanishes

$$
\begin{aligned}
Q_{n}(x \sim 0) & \sim \frac{2 m}{M_{n}} C_{n} x^{2 \beta-1} \\
2 \beta-1 & =\frac{2 \sqrt{3}}{\pi} \frac{m}{m_{0}}-1 \gg 0
\end{aligned}
$$

where the inequality is in the weak coupling limit [22]. The chiral limit and the $x \rightarrow 0,1$ limits are subtle. These observations are general, and may carry to QCD in four dimensions, although in the latter instanton effects are a strong source of chirality flips without the current mass $m$ penalty [23].

\section{The special case with $n=0$ : The would-be Goldstone mode}

In two-dimensions and finite $N_{c}$ there is no spontaneous breaking of chiral symmetry owing to Coleman theorem [24]. At large $N_{c}$ and weak coupling, chiral symmetry is almost broken due to the BKT mechanism with the appearance of an almost Golstone mode [25], the analogue of the pion and a finite chiral condensate $[22,26]$

$$
\langle\bar{\psi} \psi\rangle=-\frac{N_{c} m_{0}}{\sqrt{12}}
$$

For the would-be Goldstone or pion mode with $n=0$, $C_{0}=1$ [22] and (64) appears to obey a chiral reduction rule 
in the massless limit. Indeed, since $\bar{\psi} \psi$ is a scalar its value in a moving state is the same as its value in the state at rest. Therefore,

$$
\langle P, 0|\bar{\psi} \psi| P, 0\rangle=-2\left\langle\left[\frac{Q_{5}}{f_{0}},\left[\frac{Q_{5}}{f_{0}}, \bar{\psi} \psi\right]\right]\right\rangle=-2 \frac{\langle\bar{\psi} \psi\rangle}{f_{0}^{2}}
$$

where $Q_{5}$ is the axial $\mathrm{U}(1)$ charge operator, and $f_{0}=$ $\sqrt{N_{c} / 2 \pi}$ is the analogue of the pseudoscalar decay constant. This identification is consistent with (B5) for $n=0$ since by chiral reduction we can check that at rest

$$
\left\langle 0\left|\bar{\psi} i \gamma^{5} \psi\right| P, 0\right\rangle=-\frac{\sqrt{2}}{f_{0}}\langle\bar{\psi} \psi\rangle=\sqrt{2} f_{0}\left[\frac{m}{2} \int_{0}^{1} d x \frac{\varphi_{0}(x)}{x \bar{x}}\right]
$$

In particular, the vacuum chiral condensate is directly tied to the would-be Goldstone mode light cone wave function through

$$
\langle\bar{\psi} \psi\rangle=-\frac{1}{2} f_{0}^{2} m \int_{0}^{1} d x \frac{\varphi_{0}(x)}{x \bar{x}}
$$

We now note that the Feynman-Hellman theorem for this mode gives

$$
\frac{\partial M_{0}^{2}}{\partial m}=\langle P, 0|\bar{\psi} \psi| P, 0\rangle
$$

which together with (67) imply the Gell-Mann-OakesRenner (GOR) like relation

$$
f_{0}^{2} M_{0}^{2}=-2 m\langle\bar{\psi} \psi\rangle
$$

It follows that the gluon contribution in the traceful part of the mass in (32) is equal to the chiral symmetry breaking contribution,

$$
\left\langle P, 0\left|E^{a} E^{a}\right| P, 0\right\rangle=\langle P, 0|m \bar{\psi} \psi| P, 0\rangle=M_{0}^{2}
$$

This result is in agreement with an earlier observation made by one of us in 4-dimensions [3].

\section{Twist-four gluon parton distribution and light-cone sum rule}

Besides the quark contribution, the gluon part could also contains a regular and singular contribution [10]

$$
F_{n}(x)=\delta(x) F_{n, 0}+F_{n, \text { reg }}(x)
$$

assuming that the zeroth moment of the regular part vanishes. The occurrence of the delta-function maybe viewed as a signal of the gluon condensate on the lightfront, with the wee-gluons at $x=0$ collecting into a superfluidlike component, in an otherwise normal component of a hadron [10]. This point was recently discussed in the context of perturbation theory in [6]. We now proceed to evaluate (57) nonperturbatively in two dimensions.

In the light cone gauge with normals $n_{ \pm}^{\mu}=(1, \pm 1)$,

$$
F^{+-a}=\partial^{+} A^{-a}=n_{+}^{\mu} n_{-}^{\nu} F_{\mu \nu}^{a}=2 E^{a}
$$

the equation of motion for the gauge field simplifies to

$$
\partial^{+} E^{a}=2 g \psi_{+}^{\dagger} T^{a} \psi_{+}
$$

Inserting (75) into (57) yields

$$
\begin{aligned}
F_{n}(x)= & \frac{P_{n}^{+}}{2 M_{n}^{2}} \int \frac{d z}{2 \pi} e^{i x P_{n}^{+} z}\langle P, n|\left(\frac{2 g}{\partial^{+}} \psi_{+}^{\dagger} T^{a} \psi_{+}(0)\right) \\
& \times\left(\frac{2 g}{\partial^{+}} \psi_{+}^{\dagger} T^{a} \psi_{+}(z)\right)|P, n\rangle \\
= & \frac{P_{n}^{+}}{2 M_{n}^{2}} \int \frac{d z}{2 \pi} e^{i x P_{n}^{+} z}\langle P, n|\left(2 g \int_{0}^{\infty} d y \psi_{+}^{\dagger} T^{a} \psi_{+}(y)\right) \\
& \times\left(2 g \int_{z}^{\infty} d w \psi_{+}^{\dagger} T^{a} \psi_{+}(w)\right)|P, n\rangle
\end{aligned}
$$

Substituting the free field decomposition (21) into (76) yield 1-body (self-energy) and 2-body type contractions (cross terms). The final result is

$$
\begin{aligned}
x F_{n}(x)= & \frac{m_{0}^{2}}{4 M_{n}^{2} x}\left(\int_{\max (0, x)}^{1} d x_{1}\left(\left|\varphi_{n}\left(x_{1}\right)\right|^{2}+\left|\varphi_{n}\left(\bar{x}_{1}\right)\right|^{2}\right)\right. \\
& -\int_{\max (0, x)}^{\min (1,1+x)} d x_{1}\left(\varphi_{n}\left(x_{1}\right) \varphi_{n}^{\dagger}\left(x_{1}-x\right)\right. \\
& \left.\left.+\varphi_{n}\left(\bar{x}_{1}\right) \varphi_{n}^{\dagger}\left(\bar{x}_{1}+x\right)\right)\right) .
\end{aligned}
$$

As $x \rightarrow 0$, the self-energy and cross terms cancel with each other due to color neutrality, leading to a finite gluon distribution $x F_{n}(x)$ at $x=0$, a characteristic of color neutral bound-states. This cancellation is somehow reminiscent of the cancellation of the gauge dependence between the vertex and self-energy in the derivation of the ' $t$ Hooft equation [11]. The zeroth moment (58) reproduces the gluon-condensate (43) in agreement with the ' $t$ Hooft equation (24) and the mass sum rule (36) in the chiral limit. Notice that (43) vanishes for the pion state $\varphi_{0}(x) \sim \theta(x \bar{x})$ as it should. No singular distribution is seen to arise in (77), yet the relation (58) is satisfied.

\section{FORM FACTORS OF THE ENERGY-MOMENTUM TENSOR}

Apart from the light-cone sum rules, an empirical way to measure the gluon contribution to the mass sum rule of the nucleon, is through diffractive photo- or electro-production of heavy mesons off nucleons near threshold as suggested 
initially in [27], and pursued experimentally in [28,29]. The threshold cross section is dominated by glueball-like exchanges with tensor and scalar quantum numbers. A detailed analysis shows that the exchange probes the nucleon gravitational form factor with the glueball as a spin-2 exchange, allowing for a partial extraction of the gluon contribution to the nucleon mass [30,31]. The importance of the role of the gluons in the nucleon mass was emphasized earlier by one of us [2].

The gravitational form factor in a two-dimensional meson state is constrained by Lorentz symmetry, parity and energy-momentum conservation. Its general decomposition under these strictures leads the invariant form factors

$$
\left\langle p_{2}\left|T^{\mu \nu}\right| p_{1}\right\rangle=\frac{1}{2}\left(g^{\mu \nu} q^{2}-q^{\mu} q^{\nu}\right) \tilde{\Theta}_{1}\left(q^{2}\right)+\frac{1}{2} p^{\mu} p^{\nu} \tilde{\Theta}_{2}\left(q^{2}\right)
$$

with $q^{\mu}=p_{2}^{\mu}-p_{1}^{\mu}$ and $p^{\mu}=p_{1}^{\mu}+p_{2}^{\mu}$. Note that the transverse axial-vector $\tilde{q}^{\mu}=\epsilon^{\mu \lambda} q_{\lambda}$ does not introduce an independent 2-tensor since $\tilde{q}^{\mu} \tilde{q}^{\nu}=q^{\mu} q^{\nu}-g^{\mu \nu} q^{2}$. In 4dimensions, the two form factors correspond to the spin representations $1 \otimes 1=0 \oplus 1 \oplus 2$ with 1 excluded by parity. They reflect on the tensor exchange or graviton (2), and the scalar exchange or dilaton (0).

We now note that in 2-dimensions

$$
p^{\mu} p^{\nu}=\frac{4 M^{2}-q^{2}}{q^{2}}\left(g^{\mu \nu} q^{2}-q^{\mu} q^{\nu}\right),
$$

for on-shell momenta $p_{1,2}$. Thus, the two form factors $\widetilde{\Theta}_{1,2}$ can always be combined to a single form factor

$$
\left\langle p_{2}\left|T^{\mu \nu}\right| p_{1}\right\rangle=\frac{1}{2} p^{\mu} p^{\nu} \Theta_{2}\left(q^{2}\right) .
$$

which is consistent with the $m_{1} \otimes m_{2}=m_{1}+m_{2}$ for the irreducible representation of $U(1)$. The dilaton form factor is described by the trace

$$
\left\langle p_{2}\left|T_{\mu}^{\mu}\right| p_{1}\right\rangle=\frac{1}{2}\left(4 M^{2}-q^{2}\right) \Theta_{2}\left(q^{2}\right)
$$

The squared meson masses $M^{2}$ are fixed by the transcendental ' $t$ Hooft equation introduced before. The normalization $\Theta_{2}(0)=1$ is fixed by recalling that $H=\int d x T^{00}$ is the Hamiltonian, with $\left\langle p_{1}|H| p_{1}\right\rangle=p_{1}^{0}\left[2 p_{1}^{0}(2 \pi) \delta_{p}(0)\right]$.

The dilaton form factor is mediated by the exchange of a massive state of mass $2 M$ when recast in the form

$$
\Theta_{2}(q)=\frac{\left\langle p_{2}\left|2 T_{\mu}^{\mu}\right| p_{1}\right\rangle}{4 M^{2}-q^{2}}
$$

providing for a direct measure of the gluon content of the meson state

$$
\Theta_{2}\left(q^{2}\right)=\frac{2}{4 M^{2}-q^{2}}\left\langle p_{2}\left|\left(E^{a} E^{a}+m \bar{\psi} \psi\right)\right| p_{1}\right\rangle
$$

in the chiral limit. Near the production threshold of the mesons, the dilaton probes the trace part of the energymomentum tensor in a bound hadron in two-dimensional QCD. We interpret the $2 M$ state as a massive "longitudinalglueball" resolving into a two-meson state by mixing, for this point see also Eq. (90) and discussion below. This observation maybe useful for four dimensional QCD, where the trace part probes the trace anomaly say in a bound nucleon state, as pursued experimentally in diffractive photoproduction of heavy mesons off nucleon targets $[28,29]$. The diffractive production involves the exchange of bulk gravitons as dual of boundary massive tensor and scalar glueballs in holographic QCD [31].

\section{DUAL MASS SUM RULE}

Finally, we now show that the static electric field energy is related to the Coulomb energy represented by chirally symmetric 4-Fermi interactions. This reasoning will show how the nonperturbative aspects of the vacuum in the equal-time form are tied to a fermionic mass sum rule for the bound mesons, that is dual to the gluonic mass sum rule in the instant form.

We recall that the colored axial-vector current which is the difference between the gauge-invariant currents for left and right-handed fermions is anomalous

$$
D_{\mu}^{a b}\left(\bar{\psi} \gamma^{\mu} \gamma^{5} T^{b} \psi\right)=-\frac{g_{0}}{4 \pi} \epsilon_{\mu \nu} F^{a \mu \nu}=-\frac{g_{0}}{2 \pi} E^{a}
$$

as can be checked from the one-loop vacuum polarization diagram. Using (2) and the equation of motion for the gauge fields $D_{\mu}^{a b} F^{b \mu \nu}=-g \bar{\psi} \gamma^{\nu} T^{a} \psi$, we have

$$
\epsilon^{\mu \nu} D_{\mu}^{a b} E^{b}=-g_{0} \bar{\psi} \gamma^{\nu} T^{a} \psi=-g_{0} \epsilon^{\nu \mu} \bar{\psi} \gamma_{\mu} \gamma^{5} T^{a} \psi
$$

The last equality follows from the fact that the vector and axial-vector currents are dual in two-dimensions, $\bar{\psi} \gamma \gamma^{\nu} T^{a} \psi=-\epsilon^{\nu \mu} \bar{\psi} \gamma_{\mu} \gamma^{5} T^{a} \psi$. Therefore we have

$$
D_{\mu}^{a b} E^{b}=g_{0} \bar{\psi} \gamma_{\mu} \gamma^{5} T^{a} \psi
$$

which yields the exact covariant screening equation for the dual potential [32]

$$
\left(D^{2}+\frac{m_{0}^{2}}{2 N_{c}}\right)^{a b} E^{b}=0
$$

with the canonical mass scale $m_{0}^{2}=g_{0}^{2} N_{c} / \pi$. The emergent screening mass squared $m_{0}^{2} / 2 N_{c}$ due the light quarks is sub-leading in $1 / N_{c}$ as it should. It will be important to keep it as we now show. 
We now note the gauge invariant identity

$$
\begin{aligned}
\partial^{2}\left(E^{a} E^{a}\right) & =2\left(\partial_{\mu} E^{a}\right)\left(\partial^{\mu} E^{a}\right)+2\left(\partial^{2} E^{a}\right) E^{a} \\
& \equiv 2\left(D_{\mu}^{a b} E^{b}\right)\left(D_{\mu}^{a c} E^{c}\right)+2\left(\left(D^{2}\right)^{a b} E^{b}\right) E^{a}
\end{aligned}
$$

where in the last equality manifest gauge invariance is enforced since the starting expression $\partial^{2}\left(E^{a} E^{a}\right)$ is gauge invariant. Using (86) and (87) in (88) we obtain

$$
\left(\partial^{2}+\frac{m_{0}^{2}}{N_{c}}\right)\left(E^{a} E^{a}\right)=2 g_{0}^{2}\left(\bar{\psi} \gamma^{\mu} \gamma^{5} T^{b} \psi\right)^{2}
$$

with twice the squared screening mass observed in (87). The color anomaly fixes the gluon operator contribution in (27). The same result readily follows in the center gauge, and therefore holds for all gauges by gauge invariance. Most of the identities discussed in this section simplify for Abelian QED or the Schwinger model, thanks again to the U(1) axial anomaly. For completeness, they are quoted in Appendix A. As the first application of (89), let us derive the vacuum gluon condensate

$$
\left\langle\left(\partial^{2}+\frac{m_{0}^{2}}{N_{c}}\right)\left(E^{a} E^{a}\right)\right\rangle=\frac{m_{0}^{2}}{N_{c}}\left\langle E^{a} E^{a}\right\rangle=2 g_{0}^{2}\left\langle\left(\bar{\psi} \gamma^{\mu} \gamma^{5} T^{b} \psi\right)^{2}\right\rangle
$$

The colored 4-Fermi current-current contribution can be simplified into colorless interactions by Fierzing. Assuming vacuum factorization which is justified in large $N_{c}$ by the master field [33], we can simplify the gluon condensate to

$$
\left\langle E^{a} E^{a}\right\rangle=2 \pi\left\langle\left(\bar{\psi} \gamma^{\mu} \gamma^{5} T^{c} \psi\right)^{2}\right\rangle=\pi \frac{N_{c}^{2}-1}{N_{c}^{2}}\langle\bar{\psi} \psi\rangle^{2} \rightarrow \pi\langle\bar{\psi} \psi\rangle^{2}
$$

with the rightmost result in agreement with the large $N_{c}$ result briefly quoted in [22]. The fact that the quark and gluon condensates are tied in two dimensions, is reminiscent of the way both condensates are tied to the instanton packing fraction in four dimensions $[34,35]$.

We now use (89) to characterize a dual mass sum rule for the bound mesons. The gluon mass operator in Eq. (32) can be unwound in terms of 4-Fermi colored interactions much like in the vacuum in (91), with the result

$$
\begin{aligned}
M_{n}^{2}= & 2 \pi\langle P, n|(\bar{\psi} \psi)^{2}+\left(\bar{\psi} i \gamma^{5} \psi\right)^{2} \\
& -\frac{2}{N_{c}}\left(\bar{\psi} \gamma^{\mu} \psi\right)^{2}|P, n\rangle+\frac{1}{2}\langle P, n|m \bar{\psi} \psi| P, n\rangle
\end{aligned}
$$

after Fierz rearrangement. In two-dimensions, the gluon field is longitudinal and sourced by the quarks in the bound state. The fermionic mass sum rule (92) is dual to the gluonic mass sum rule (54), and it is somehow illusive to try to distinguish one from the other. This is reminiscent of the Coulomb energy between a pair of classical charges which can be either assessed using (screened) Coulomb law between the charges, or the energy stored in the (screened) Coulomb field surrounding the charges. In most of the above discussions, this should be kept in mind.

We note that (92) is how the meson masses are generated in models that capture chiral symmetry breaking without confinement, such as the instanton model for the QCD vacuum [34,35], and bound state models [35-37] in four dimensions. For the former, the strong instanton and antiinstanton fields are able to trap quarks into left- and righthanded zero mode states, thanks to the axial U(1) anomaly, and generate quasilocal 4-Fermi determinantal interactions. The latter are at the origin of finite constituent masses and meson masses much like (92). It is remarkable, that in two dimensions and large $N_{c}$, the subleading quantum screening of the electric field as enforced by the colored axialvector anomaly (84), is also at the origin of the leading chirally symmetric and local 4-Fermi interactions, with a dual mass relation for the bound mesons in any frame. In a way, the spontaneous breaking of chiral symmetry appears to be the chief nonperturbative mechanism for mass generation for the light hadrons, in a confining theory such as QCD where the string breaks through quantum screening by light quarks.

\section{CONCLUSIONS}

We have explored the role of the chiral and gluon matrix elements in the composition of the mass of the meson bound states in two dimensional QCD in the ' $t$ Hooft limit. Both the chiral and gluon matrix elements in a bound meson are probed by the chiral-odd quark parton distribution, and the gluon parton distribution respectively. We have explicitly derived these distributions in the ' $t$ Hooft limit. Their zeroth moments are indeed related to the quark and gluon condensate in the meson states. For the chiral odd quark distribution, we have shown that the lowest moment arises not from a delta-function accumulation of wee partons at the edge or $x \sim 0$, but rather from their rapid and specific depletion at the edge. For the gluon distribution, the situation is more subtle. Color neutrality forces the gluon distribution $x F_{n}(x)$ to be finite as $x \rightarrow 0$ without the occurrence of a singular contribution, yet the mass sum rule in the chiral limit is fulfilled. Our use of the PV prescription does not upset the light front sum rules in leading order in $1 / N_{c}$.

The form factor of the energy-momentum tensor splits into two invariant form factors, one for the graviton or tensor coupling, and the other for the dilaton or scalar coupling. However, in two dimensions they are related. The traceful part is similar to that in four dimensional QCD, although for different reasons since two-dimensional QCD is nonconformal. The traceful part of the energy 
momentum tensor fixes the bound meson masses, and dominates the gravitational form factor. Finally but remarkably, a dual mass sum rule is shown to emerge solely in terms of chirally invariant 4-Fermi interactions.

The present results may be specific to two dimensions, since QCD is nonconformal. Also, in the light cone gauge, the structure of the light cone zero modes arising from a perturbative treatment, is regulated by quantum mechanics in the absence of the transverse degrees of freedom. In four dimensions these zero modes are regulated by field theory, and may be at the origin of the singular contributions in some higher twist contributions to the parton distribution functions, as we discussed. More work in this direction is needed.

\section{ACKNOWLEDGMENTS}

This work was supported by the U.S. Department of Energy under Contract No. DE-SC0020682 for X. J. and No. DE-FG-88ER40388 for I. Z.

\section{APPENDIX A: MASSIVE ABELIAN QED}

The analysis in Sec. VI carries to two dimensional massive QED with more simplifications. The U(1) axial anomaly Abelianizes

$$
\partial_{\mu}\left(\bar{\psi} \gamma^{\mu} \gamma^{5} \psi\right)=\frac{e}{2 \pi} \epsilon_{\mu \nu} F^{\mu \nu}=\frac{e}{\pi} E
$$

which is again given by the one-loop diagram. Following the same reasoning as given earlier, we have for Maxwell equation

$$
\partial_{\mu} F^{\mu \nu}=-e \bar{\psi} \gamma^{\nu} \psi
$$

and using the duality of the currents

$$
\partial_{\mu} E=-e \bar{\psi} \gamma \gamma_{\mu} \gamma^{5} \psi
$$

This shows that the pseudo-potential $E$ describes a massive free boson

$$
\left(\partial^{2}+\frac{e^{2}}{\pi}\right) E=0
$$

with $E^{2}$ obeying the screened source equation

$$
\left(\partial^{2}+\frac{2 e^{2}}{\pi}\right) E^{2}=2 e^{2}\left(\bar{\psi} \gamma^{\mu} \gamma^{5} \psi\right)^{2}=-2 e^{2}\left(\bar{\psi} \gamma^{\mu} \psi\right)^{2}
$$

with again twice the squared screening mass. In the massive case, the squared meson mass obeys the analogue of the sum rule (36), namely

$$
\begin{aligned}
M^{2} & =\frac{1}{2}\left\langle P\left|E^{2}\right| P\right\rangle+\frac{1}{2}\langle P|m \bar{\psi} \psi| P\rangle \\
& =\frac{\pi}{2}\left\langle P\left|\left(\bar{\psi} \gamma^{\mu} \gamma^{5} \psi\right)^{2}\right| P\right\rangle+\frac{1}{2}\langle P|m \bar{\psi} \psi| P\rangle .
\end{aligned}
$$

In two dimensions the currents bosonize

$$
\bar{\psi} i \gamma^{\mu} \gamma^{5} \psi=\frac{1}{\sqrt{\pi}} \partial^{\mu} \phi \quad \bar{\psi} \gamma^{\mu} \psi=-\frac{1}{\sqrt{\pi}} \epsilon^{\mu \nu} \partial_{\nu} \phi .
$$

In the massless case $\phi$ is a free massive boson with squared mass $M^{2}=e^{2} / \pi$, which is seen to be satisfied by (A6)

$$
\begin{aligned}
M^{2} \rightarrow \frac{1}{2}\left\langle P\left|E^{2}\right| P\right\rangle & =-\frac{\pi}{2(\sqrt{\pi})^{2}}\left\langle P\left|\left(\partial_{\mu} \phi\right)^{2}\right| P\right\rangle \\
& =M^{2}\left[\frac{1}{2}\left\langle P\left|\phi^{2}\right| P\right\rangle=1\right] .
\end{aligned}
$$

The last bracket as a scalar is frame invariant. We evaluated it in the meson rest frame, using the equal-time free field decomposition

$$
\phi(x)=\int \frac{d k}{2 \pi} \frac{1}{\sqrt{E_{k}}}\left(e^{-i k \cdot x} \alpha(k)+e^{i k \cdot x} \alpha^{\dagger}(k)\right)
$$

and the canonical rules $\left[\alpha(k), \alpha^{\dagger}(p)\right]=2 \pi \delta(k-p)$. The meson state with scattering normalization is defined as $|P\rangle=\sqrt{P^{0}}\left(\alpha^{\dagger}(P)|0\rangle\right)$ with $P^{0}=E_{P}=M$ at rest.

\section{APPENDIX B: BEHAVIOR OF LIGHT-FRONT WAVE FUNCTION NEAR THE EDGES IN THE CHIRAL LIMIT}

The wave functions solution to the ' $t$ Hooft equation Eq. (24), describe the quark parton distribution amplitudes on the light cone

$\varphi_{n}(x)=\frac{1}{f_{0}} \int \frac{d z}{2 \pi} e^{i x P^{+} z}\left\langle 0\left|\bar{\psi}_{+}(0)[0, z] \psi_{+}(z)\right| P, n\right\rangle$

in the gauge $A_{-}=0$, with $[0, z]=1$. They satisfy conjugate symmetry $\varphi_{n}(1-x)=(-1)^{n} \varphi_{n}(x)$ along with a number of integral identities [38]. They are orthonormal

$$
\int_{0}^{1} d x \varphi_{n}^{*}(x) \varphi_{n}(x)=\delta_{n m}
$$

and vanish at the edges

$$
\varphi_{n}(x \sim 0,1) \sim C_{n}(x \bar{x})^{\beta}
$$

with $\beta$ solution to

$$
\pi \beta \cot (\pi \beta)=\frac{\beta^{2}}{m_{0}^{2}}-\frac{m^{2}}{m_{0}^{2}}
$$


which for small masses give $\beta \sim \sqrt{3} m / \pi m_{0}$. Away from the edges, the solutions are not known analytically except for the almost Goldstone mode with $M_{0} \sim \mathcal{O}(m), C_{0}=1$ and $\varphi_{0}(x)=\theta(x \bar{x})$ (except at the edges) [22]. In general, the exact solutions to (24) are only known numerically. For large $n$, the solutions follow analytically from semiclassics with $\varphi_{n}(x) \sim \sqrt{2} \sin \left((n+1) \pi x+\delta_{n}(x)\right)$ (except at the edges) and $M_{n}^{2} \approx n \pi^{2} m_{0}^{2}[11,38]$. The pseudoscalar transition amplitude

$$
\left\langle 0\left|\bar{\psi} i \gamma^{5} \psi\right| P, n\right\rangle=\sqrt{2} f_{0}\left[\frac{m}{2} \int_{0}^{1} d x \frac{\varphi_{n}(x)}{x \bar{x}}\right]
$$

fixes the decay constant $f_{0}=\left(N_{c} / 2 \pi\right)^{\frac{1}{2}}$.

\section{APPENDIX C: VIRIAL THEOREM}

In this Appendix we provide a simple derivation of the virial theorem $\mathcal{K}=\mathcal{G}$ established in Eq. (39). For that, consider the rescaling of the only dimensionful parameters $g_{0}^{2}=M^{2} \lambda_{1}$ and $m=M \lambda_{2}$ with $M$ being a common mass parameter and $\lambda_{1}, \lambda_{2}$ being massless. All meson masses are proportional to $M$ by dimensions, i.e., $M_{n}=M f\left(\lambda_{1}, \lambda_{2}\right)$. Using the Feynman-Hellman theorem

$$
M_{n}=\left\langle M \frac{\partial H}{\partial M}\right\rangle
$$

we have

$$
M_{n}=\mathcal{M}+2 \mathcal{G}
$$

since the gluon energy in the Hamiltonian (5) is propagational to $g_{0}^{2} \propto M^{2}$ and the mass term is proportional to $m \propto M$. By comparing with the mass decomposition,

$$
M_{n}=\mathcal{M}+\mathcal{G}+\mathcal{K}
$$

we found

$$
\mathcal{G}=\mathcal{K},
$$

which is the desired virial theorem.

In the large- $N_{c}$ limit, the Hamiltonian in Eq. (5) can be expressed through bosonization in terms of meson operators [12]. The Bars-Green equation arises as one tries to diagonalize the Hamiltonian. By applying the virial theorem to the bosonized form of the Hamiltonian and then expressing in terms of the components, one obtains the desired virial theorem expressed in terms of the Bars-Green wave functions.
[1] R. Abdul Khalek et al., EIC yellow report, arXiv:2103 .05419 .

[2] X.-D. Ji, Phys. Rev. Lett. 74, 1071 (1995).

[3] X.-D. Ji, Phys. Rev. D 52, 271 (1995).

[4] A. Metz, B. Pasquini, and S. Rodini, Phys. Rev. D 102, 114042 (2020).

[5] O. Gryniuk, S. Joosten, Z.-E. Meziani, and M. Vanderhaeghen, Phys. Rev. D 102, 014016 (2020).

[6] Y. Hatta and Y. Zhao, Phys. Rev. D 102, 034004 (2020).

[7] S. Borsanyi, Z. Fodor, C. Hoelbling, L. Lellouch, K. Szabo, C. Torrero, and L. Varnhorst, arXiv:2007.03319.

[8] F. Zeng, X.-Y. Wang, L. Zhang, Y.-P. Xie, R. Wang, and X. Chen, Eur. Phys. J. C 80, 1027 (2020).

[9] Y. Chen, arXiv:2010.00900.

[10] X. Ji, Nucl. Phys. B960, 115181 (2020).

[11] G. 't Hooft, Nucl. Phys. B75, 461 (1974).

[12] Y. Jia, S. Liang, X. Xiong, and R. Yu, Phys. Rev. D 98, 054011 (2018).

[13] X. Ji, Y. Liu, and I. Zahed, Phys. Rev. D 99, 054008 (2019).

[14] I. Bars and M. B. Green, Phys. Rev. D 17, 537 (1978).

[15] W. Lucha and F. F. Schoberl, Phys. Rev. Lett. 64, 2733 (1990).

[16] Y. Jia, R. Yu, and X. Xiong, Phys. Rev. D 98, 074024 (2018).
[17] A. Efremov and P. Schweitzer, J. High Energy Phys. 08 (2003) 006.

[18] F. Aslan and M. Burkardt, Phys. Rev. D 101, 016010 (2020).

[19] S. Bhattacharya, K. Cichy, M. Constantinou, A. Metz, A. Scapellato, and F. Steffens, Phys. Rev. D 102, 114025 (2020).

[20] W. Weisberger, Phys. Rev. D 5, 2600 (1972).

[21] S. J. Brodsky, F. J. Llanes-Estrada, and A. P. Szczepaniak, eConf C070910, 149 (2007).

[22] A. Zhitnitsky, Sov. J. Nucl. Phys. 43, 999 (1986).

[23] E. Shuryak and I. Zahed, Phys. Rev. D 103, 054028 (2021).

[24] S. R. Coleman, Commun. Math. Phys. 31, 259 (1973).

[25] E. Witten, Nucl. Phys. B145, 110 (1978).

[26] M. Burkardt, in Workshop on Quantum Infrared Physics, edited by H. Fried and B. Muller (World Scientific, 1995).

[27] D. Kharzeev, H. Satz, A. Syamtomov, and G. Zinovjev, Eur. Phys. J. C 9, 459 (1999).

[28] K. Hafidi, S. Joosten, Z. Meziani, and J. Qiu, Few Body Syst. 58, 141 (2017).

[29] A. Ali et al. (GlueX Collaboration), Phys. Rev. Lett. 123, 072001 (2019).

[30] Y. Hatta and D.-L. Yang, Phys. Rev. D 98, 074003 (2018). 
[31] K. A. Mamo and I. Zahed, Phys. Rev. D 101, 086003 (2020).

[32] L. Belvedere, K. Rothe, B. Schroer, and J. Swieca, Nucl. Phys. B153, 112 (1979).

[33] E. Witten, NATO Sci. Ser. B 59, 403 (1980).

[34] T. Schäfer and E. V. Shuryak, Rev. Mod. Phys. 70, 323 (1998).

[35] M. A. Nowak, M. Rho, and I. Zahed, Chiral Nuclear Dynamics (World Scientific, 1996).
[36] A. Bashir, L. Chang, I. C. Cloet, B. El-Bennich, Y.-X. Liu, C. D. Roberts, and P. C. Tandy, Commun. Theor. Phys. 58, 79 (2012).

[37] G. Eichmann, H. Sanchis-Alepuz, R. Williams, R. Alkofer, and C. S. Fischer, Prog. Part. Nucl. Phys. 91, 1 (2016).

[38] C. G. Callan, Jr., N. Coote, and D. J. Gross, Phys. Rev. D 13, 1649 (1976). 